Review Article

\title{
Tendon Stem/Progenitor Cells and Their Interactions with Extracellular Matrix and Mechanical Loading
}

\author{
Chuanxin Zhang, ${ }^{1}$ Jun Zhu, ${ }^{1}$ Yiqin Zhou $\mathbb{D}^{1},{ }^{1}$ Bhavani P. Thampatty, \\ and James H-C. Wang $\mathbb{D}^{2}$ \\ ${ }^{1}$ Joint Surgery and Sports Medicine Department, Shanghai Changzheng Hospital, Second Military Medical University, \\ Shanghai, China \\ ${ }^{2}$ MechanoBiology Laboratory, Departments of Orthopaedic Surgery, Bioengineering, and Physical Medicine and Rehabilitation, \\ University of Pittsburgh, Pittsburgh, Pennsylvania, USA
}

Correspondence should be addressed to James H-C. Wang; wanghc@pitt.edu

Received 21 May 2019; Revised 4 August 2019; Accepted 17 August 2019; Published 13 October 2019

Guest Editor: Jun Li

Copyright (c) 2019 Chuanxin Zhang et al. This is an open access article distributed under the Creative Commons Attribution License, which permits unrestricted use, distribution, and reproduction in any medium, provided the original work is properly cited.

Tendons are unique connective tissues in the sense that their biological properties are largely determined by their tendon-specific stem cells, extracellular matrix (ECM) surrounding the stem cells, mechanical loading conditions placed on the tendon, and the complex interactions among them. This review is aimed at providing an overview of recent advances in the identification and characterization of tendon stem/progenitor cells (TSPCs) and their interactions with ECM and mechanical loading. In addition, the effects of such interactions on the maintenance of tendon homeostasis and the initiation of tendon pathological conditions are discussed. Moreover, the challenges in further investigations of TSPC mechanobiology in vitro and in vivo are outlined. Finally, future research efforts are suggested, which include using specific gene knockout models and single-cell transcription profiling to enable a broad and deep understanding of the physiology and pathophysiology of tendons.

\section{Introduction}

Tendons are specialized tissues that enable joint movements by transmitting muscular forces from muscle to bone. They are relatively hypocellular tissues that are composed of an extracellular matrix (ECM), predominantly of collagen [1, 2], which is organized in a hierarchical manner. The collagen molecules assemble into fibrils that form fibers, fibers form fascicles, and bundles of fascicles form the fascicular matrix (FM). Endotenon, also known as the interfascicular matrix (IFM), occupies the space between fascicle bundles and is covered by epitenon and another layer of paratenon forming the whole tendon unit $[1,3]$. Additionally, tendon contains two major types of cells, tenocytes and tendon stem/progenitor cells (TSPCs). Under normal conditions, tenocytes are responsible for maintaining tendon homeostasis, whereas TSPCs replenish tendon cells by undergoing self-renewal and differentiation $[4,5]$. Tendon also contains other cell types such as endothelial cells, synovial cells of the tendon sheaths, and chondrocytes at the pressure and insertion sites in smaller amounts $[3,6]$.

The first study that isolated and characterized TSPCs indicates that these cell populations reside within the tendon proper (midsubstance) that comprises FM and IFM [4]. These cells are not strictly classified as "stem" cells, since they display heterogeneity in their biological properties. Instead, they were classified as "stem/progenitor" cells considering the possibility of inclusion of progenitor cells, which are destined to undergo differentiation towards a specific lineage. Indeed, while TSPCs possess multidifferentiation potential, they may contain progenitors that may specifically differentiate into tenocytes. Although TSPCs have been isolated and identified more than a decade ago, the lack of specific markers poses a challenge to study them further. In addition, distinctive populations of TSPCs have been identified from locations other than the tendon proper 
such as peritenon [7-9], but their functions are yet to be defined.

The hierarchical tendon structure is well optimized for its specific functions. Mechanical loads placed on the tendons are transformed into biochemical signals to tendon cells, which respond appropriately to regulate the metabolism of tendon and its structural properties $[10,11]$. However, mechanical overloading may cause tendon injury, which is a common clinical problem affecting the quality of life for millions [12, 13]. Once tendon injury occurs, a successive natural healing process is thought to take place in three phases: inflammation (infiltration of inflammatory cells), proliferation (formation of new cells), and remodeling of ECM (change in the structure and form of tendon matrix) [14].

There are two categories of tendon injury: acute and chronic. Acute tendon injury, either partial or complete tear, results from a sudden tendon rupture that may be spontaneous or caused by direct trauma. Chronic tendon injury, commonly referred to as tendinopathy, is generally thought to result from repetitive mechanical overloading on the tendon, genetic predisposition, and age-related degeneration [15-17]. While pain and disability are the clinical indicators, the pathological features of tendinopathy include changes in the extracellular matrix (ECM) with collagen disorganization, proteoglycan deposition, neovascularization, and calcification [18, 19]. Several mechanisms have been proposed for the pathogenesis of tendinopathy that include with or without inflammation-mediated changes in tendon [20-22].

Due to the hypocellularity and hypovascularity of the tendon, the natural healing ability of tendons is rather limited [23, 24]. Moreover, tendon healing results in the formation of scar tissues, manifested by disorganized collagen matrix, increased proteoglycan and glycosaminoglycan content, and increased noncollagenous ECM [25-27]. Despite years of research, restoration of damaged tendon tissues to normal structure and function remains a great challenge in sports medicine and orthopaedic surgery. In particular, tendinopathic tendons respond poorly to current treatments including NSAIDS, corticosteroid and PRP injections, exercise-based physical therapy, and surgery [28]. Although heavy slow resistance (HSR) training reduces pain and improves collagen fibril morphology in a small number of patients [29], the efficacy of HSR training remains to be verified with large randomized controlled trials. By and large, current therapeutic strategies are palliative due to the limited understanding of the cellular and molecular mechanisms of tendinopathy. The development of new effective treatment options needs an in-depth understanding of basic tendon biology and, in particular, the function of tendon cells and their interactions with ECM in tendon.

Moreover, tendon is a mechanoresponsive tissue. Therefore, tendon homeostasis is maintained not only by the cells and ECM, but also by the mechanical loads placed on the tendon. TSPCs are responsive to mechanical loading, and some findings suggest that TSPCs are likely responsible for the development of degenerative tendinopathy by virtue of their multidifferentiation potential to nontenocyte phenotypes under excessive mechanical loading conditions [30-33]. Considering the emerging role of TSPCs in tendon homeostasis and in the development of tendon's pathological condi- tions, and their potential applications in tissue engineering of injured tendons, a deeper understanding of the interactions between TSPCs, ECM, and mechanical loading is essential. In this review, we discuss the efforts to identify and characterize TSPCs with regard to their locations in tendon. We also provide an overview of the interactions between TSPCs, ECM, and mechanical loading that may be important advances in tendon biology and pathology. Finally, we discuss the challenges in understanding TSPC biology and provide our perspectives on future research directions.

\section{Tendon Cells}

The cell populations in tendon are heterogeneous, and they are identified based on their anatomical locations such as FM, IFM, and paratenon, as well as the perivascular area close to paratenon in and around the tendon [34]. Still, there is little understanding of the phenotypical differences between these cell populations and specific markers to discriminate between them. The primary cell type in tendon is tenocytes, which are elongated fibroblast-like cells with spindle-shaped nuclei that are found mainly in FM [35]. Commonly used markers for tenocytes are collagen types I and III and tenomodulin (TNMD) [36]. It should be noted, however, that the term tenocyte in literature can be somewhat "arbitrary," meaning that some so-called tenocytes are likely stem/progenitor cells.

Until the discovery of TSPCs, tenocytes were thought to be the only major cell type in tendon. The quest for the presence of adult stem cells in tendons began with two previous observations: (a) human and mouse tendons develop fibrocartilage and ossification in response to injury and (b) tendon-derived immortalized cell lines and human tendonderived "fibroblasts" possess multidifferentiation capabilities in vitro $[24,37,38]$. Before long, TSPCs were first identified in humans and mice in 2007 [4]. The TSPCs isolated from the tendon proper with stem cell characteristics of clonogenicity, multipotency, and self-renewal could regenerate tendonlike tissues after in vitro expansion and in vivo transplantation [4]. In addition, an ECM-rich niche composed of biglycan and fibromodulin controls the self-renewal and differentiation of TSPCs [4]. Shortly, two other groups isolated and identified this unique stem cell population from tendons of rabbits and rats and characterized them extensively $[5,39]$. In these studies, TSPC colonies exhibit large variations in cell proliferation and differentiation possibly due to differences in species, tissue origin, and initial seeding density in culture. The shape of TSPCs also varies between species, tissue origin, cell passages, and confluence of the culture [40]. Additionally, the success of obtaining a large pool of TSPCs depends on the age of the animal/individual; aged tendon tissues are depleted of at least 70\% TSPCs, they proliferate much slower than young TSPCs, and they have much lower expression of stem cell markers [41].

TSPCs possess distinct properties compared to resident tenocytes. They differ from tenocytes in many aspects such as shape, proliferation and differentiation potential, and expression of stem cell-specific markers [5]. Rabbit TSPCs are more cobblestone-shaped with large nuclei, while 
tenocytes are more elongated, fibroblast-like with small nuclei in culture. Overall, TSPCs also proliferate much faster than tenocytes in culture [5]. Moreover, the capacity of multidifferentiation potency allows TSPCs to differentiate into tenocytes as well as nontenocytes, including adipocytes, chondrocytes, and osteocytes $[4,5,39]$. While both express common tendon-related markers including collagen type I, collagen type III, tenascin C, and TNMD, TSPCs in vitro express stem cell markers such as Oct-4, SSEA-1/4 and nucleostemin, while tenocytes exhibit a minimal expression of these markers [5].

TSPCs and bone marrow mesenchymal stem cells (BMSCs) share many of the same markers, yet the expression pattern is not identical between humans, mice, and rats [4, 41]. For example, human and mouse TSPCs lack CD18, but it is expressed in human BMSCs, and human and rat TSPCs do not express CD106, while it is expressed in human and mouse BMSCs $[4,39,42,43]$. Also, over $60 \%$ of mouse TSPCs express CD90.2 whereas mouse BMSCs lack the expression [4]. Compared to mouse BMSCs, mouse TSPCs express higher mRNA levels of scleraxis (Scx), Comp, SOX9, and Runx2. Human TSPCs also express higher levels of TNMD than human BMSCs [4]. Moreover, rat TSPCs have higher mRNA expression of tenogenic, adipogenic, and osteogenic markers compared to rat BMSCs at basal level [44]. These differences between species could suggest that TSPCs and BMSCs represent different developmental stages of a common MSC predecessor. Finally, since TSPCs tend to differentiate into tendon-specific cells (tenocytes) compared to BMSCs, whereas BMSCs tend to differentiate towards osteogenic lineage $[4,45]$, TSPCs may be ideal cells for tissue engineering of injured tendons.

\section{Locations of TSPCs}

The exact location of TSPCs in tendon is unclear. The IFM is a suggested location based on several observations. First of all, in the pioneering studies, TSPCs were isolated and characterized from the tendon proper after stripping off the tendon sheath and surrounding paratenon possibly to exclude vascular cells from the peritenon region $[4,5,39]$, indicating IFM as a potential source of TSPCs. This speculation is strengthened by the observation that there are morphological and metabolic differences between IFM and FM. The IFM region is highly cellular and more vascular and has a fast turnover of noncollagenous matrix compared to FM, and the cells within IFM are round in shape compared to elongated tenocytes in FM (Figure 1) [46, 47]. However, tendon healing is thought to result from cells originating from multiple locations [48]. Therefore, it is possible that TSPCs may exist within each region in tendon and they may differ from one region to another, in terms of origins of progenitors, numbers of progenitor cells, and differentiation potentials. In fact, TSPCs have been isolated from peritenon/perivascular sources and their stem cell properties, such as clonogenicity, multipotency, and surface marker expression, have been determined and compared with TSPCs from the tendon proper (Table 1).
The perivascular area is an important source of stem/progenitor cells in tendon. Cells in intact human supraspinatus tendon biopsies and perivascular cells isolated from the microvessels of the same biopsies have been characterized. The results suggest that the perivascular region is a source of tendon precursor cells [7]. These cells express classical stem cell markers musashi-1, nestin, prominin-1/CD133, CD29, and CD44 as well as tendon-specific markers Scx and Smad 8. They also retain stem cell characteristics in culture. Later on, another study characterized TSPCs from the peritenon and tendon proper of mouse Achilles tendons [8]. Cells derived from the peritenon form less stem/progenitor cell colonies relative to those from the tendon proper. Analysis of surface markers for TSPCs from both regions indicated that they are $\mathrm{Scal}^{+}$(stem cell marker), $\mathrm{CD}^{+} 0^{+}$, and $\mathrm{CD} 44^{+}$(fibroblast markers) (Table 1).

Progenitors from both the tendon proper and the peritenon demonstrate a low percentage of cells positive for leukocytic, hematopoietic, and perivascular markers CD18, CD34, and $\mathrm{CD} 133$, indicative of subpopulations of progenitor cells with stem cell properties, fibroblast features, and little contribution from leukocytic, hematopoietic, or perivascular sources. The marker profile of TSPCs isolated from the tendon proper is consistent with that described by Bi et al. [4]. Tendon proper stem/progenitor cells express high levels of TNMD and Scx, indicative of enrichment of stem/progenitor cells of a tendon origin. In contrast, cells of the peritenon demonstrate relative increases in the expression of vascular (endomucin) and pericyte (CD133) markers relative to cells from the tendon proper. However, cells from both regions were able to form primitive tendon constructs when seeded within a fibrin gel. These tendon constructs displayed tendon-like characteristics such as the expression of collagen type I and TNMD and formation of collagen fibril and fiber along the long axis. One particular distinction noted between the progenitors from the two sources was that when these cells were grown in osteogenic media, only progenitors from the tendon proper deposited calcium within the cell layer. This feature may provide an explanation for the calcification and ossification, which is a typical feature of tendinopathy in the tendon proper. Recently, transcriptome profiles of isolated murine Achilles tendon proper- and peritenonderived progenitor cells were carried out [49]. It was found that progenitor cells from the tendon proper differ from peritenon progenitor cells in the differential expression of genes, including Scx, Mohawk, Thbs4, and Wnt10 $\alpha$. The distinct types of TSPCs within the tendon proper and the peritenon may differentially contribute to intrinsic (tendon proper) and extrinsic (epitenon and paratenon) tendon repair mechanisms. The intrinsic repair may require those progenitor cells that predominantly express tendon markers, while extrinsic repair may involve those stem cells recruited from the perivascular area.

To understand the location of tendon stem/progenitor cells in tendons and their role in tendon repair, in vivo identity of TSPCs and their role in tendon healing have been investigated in rats using the IdU label-retaining method [9]. The results showed that label-retaining cells (LRCs) could be identified at the tendon proper, peritenon, and 


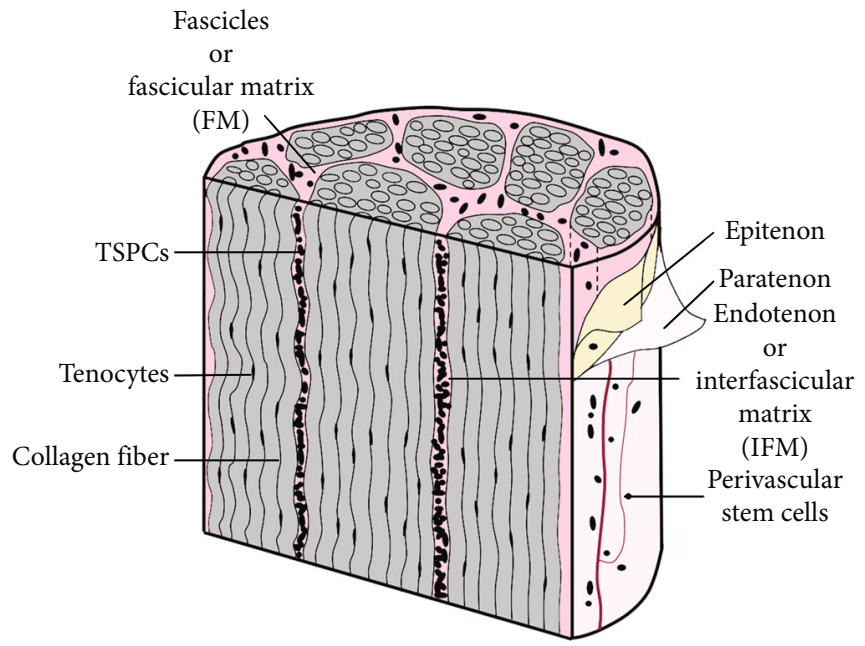

FIGURE 1: A simplified model of tendon structure adapted from Speisz et al. [47] showing fascicular matrix (FM), interfascicular matrix (IFM), and paratenon. The elongated tenocytes are located in FM in between fibers. The TSPCs from the tendon proper are presumably located in IFM; its exact location is yet to be determined, however.

TABLE 1: Sources and properties of TSPCs.

\begin{tabular}{|c|c|c|c|}
\hline Sources & Properties & Markers & Reference \\
\hline \multirow{4}{*}{$\begin{array}{l}\text { Tendon } \\
\text { proper/midsubstance }\end{array}$} & $\begin{array}{l}\text { (i) Typical stem cell characteristics } \\
\text { (ii) Multipotent } \\
\text { (iii) Distinct from BMSCs }\end{array}$ & $\begin{array}{l}\text { Scleraxis, TNMD, collagen type I, } \\
\text { tenascin, Sca1, CD90.2, CD44, CD146, } \\
\text { Oct-4, SSEA-4, nucleostemin }\end{array}$ & $\begin{array}{l}\text { Bi et al., } 2007 \text { [4]; Zhang } \\
\text { and Wang, } 2010 \text { [5]; Rui } \\
\text { et al., } 2010 \text { [39] }\end{array}$ \\
\hline & $\begin{array}{l}\text { (i) Typical stem cell characteristics } \\
\text { (ii) Form tendon constructs that express } \\
\text { collagen type I and TNMD } \\
\text { (iii) Deposit calcium in osteogenic medium } \\
\text { (iv) Potential nonvascular origin }\end{array}$ & $\begin{array}{l}\text { Sca1, CD90, CD44, CD19, CD34, CD13, } \\
\text { musashi-1, TNMD, scleraxis }\end{array}$ & $\begin{array}{c}\text { Mienaltowski et al., } 2013 \\
{[8]}\end{array}$ \\
\hline & $\begin{array}{l}\text { (i) Typical stem cell characteristics } \\
\text { (ii) Potential nonvascular origin }\end{array}$ & $\begin{array}{l}\text { CD146, Oct-4, nanog, SOX-2, } \\
\text { nucleostemin }\end{array}$ & Tan et al., 2013 [9] \\
\hline & $\begin{array}{l}\text { (i) Typical stem cell characteristics } \\
\text { (ii) Higher self-renewal and tenogenesis } \\
\text { capacity, larger collagen fibril diameter } \\
\text { compared to nestin-negative TSPCs }\end{array}$ & $\begin{array}{l}\text { Nestin, CD146, CD90, CD44, CD105, } \\
\text { CD51 }\end{array}$ & Yin et al., 2016 [52] \\
\hline \multirow{4}{*}{ Peritenon } & $\begin{array}{l}\text { (i) Express classical stem cell markers } \\
\text { (ii) Retain stem cell characteristics in culture } \\
\text { (iii) Potential vascular origin }\end{array}$ & $\begin{array}{l}\text { Musashi-1, nestin, prominin-1/CD133, } \\
\text { nestin, collagen types I and III, Smad8, } \\
\text { CD29, CD44, scleraxis }\end{array}$ & Tempfer et al., 2009 [7] \\
\hline & $\begin{array}{l}\text { (i) Typical stem cell characteristics } \\
\text { (ii) Display TSPC surface profile } \\
\text { (iii) Form tendon constructs that express } \\
\text { collagen type I and TNMD } \\
\text { (iv) Potential vascular origin }\end{array}$ & $\begin{array}{c}\text { Sca1, CD90, CD44, CD19, CD34, CD13, } \\
\text { musashi-1 }\end{array}$ & $\begin{array}{c}\text { Mienaltowski et al., } 2013 \\
{[8]}\end{array}$ \\
\hline & (v) Both vascular and nonvascular sources & $\begin{array}{l}\text { CD146, Oct-4, Nanog, SOX-2, } \\
\text { nucleostemin }\end{array}$ & Tan et al., 2013 [9] \\
\hline & $\begin{array}{l}\text { (i) Typical stem cell characteristics } \\
\text { (ii) Neural crest-like stem cells } \\
\text { (iii) Potential vascular origin } \\
\text { (iv) Involved in tendon repair }\end{array}$ & $\begin{array}{l}\text { CD29, CD90, P75, vimentin, Snail, SOX- } \\
10\end{array}$ & Xu et al., 2015 [50] \\
\hline
\end{tabular}

tendon-bone junction. Most of the TSPCs isolated from the tendon proper were LRCs suggesting that LRCs were likely to be TSPCs isolated from tendon tissue. Most of the LRCs were found to be embedded between parallel collagen fibers; however, some LRCs were also found at the perivascular region at the peritenon, and these LRCs expressed CD146. Isolated TSPCs also expressed CD146 initially, but lost its expression during the in vitro expansion, although they still expressed Nanog, Oct-4, SOX-2, and nucleostemin. In the tendon injury model with a window defect, the LRCs migrated, 
proliferated, and activated for tenogenesis in the wound [9]. In another study, a subpopulation of cells exhibiting stem characteristics of clonogenicity, multipotency, and self-renewal capacity putatively of perivascular origin that reside within rat peritenon has been identified [50]. These cells expressed markers P75 (neurotrophin receptor), vimentin, SOX-10, and Snail consistent with neural crest stem cells (NCSCs). In the event of tendon injury, these perivascular cells may migrate from the vessels to interstitial space, and produce collagenous and noncollagenous proteins to repair damaged ECM [51].

The molecular profiling of individual cells derived from tendon identified a distinct subpopulation of nestin ${ }^{+}$cells that express stem cell markers (CD146, CD105, etc.) and tenolineage markers ( $\mathrm{Col} \mathrm{I}$, tenascin $\mathrm{C}$, etc.) that are likely to be TSPCs [52]. Nestin is a type IV filament protein expressed in a variety of adult stem/progenitor cell populations that is required for the proper self-renewal [53-55]. Analysis of phenotypic differences between nestin ${ }^{+}$TSPCs isolated in vitro from the tendon proper of human Achilles tendon shows a better tenogenic potential and selfrenewing capacity and larger collagen fibril diameter than nestin ${ }^{-}$TSPCs (Table 1). The nestin expression seems to be essential for the tenogenesis of TSPCs since the expression of nestin led to a strong induction of Scx and Mkx and tendon-related marker genes elastin and collagen type I and XIV. However, both nestin ${ }^{+}$and nestin ${ }^{-}$TSPCs display a similar propensity to differentiate into osteocytes, adipocytes, and chondrocytes and have similar proliferation potential. Nestin knockdown significantly reduces colony forming capacity and causes the loss of the typical shape of TSPCs. Nestin knockdown also impairs tendon repair and regeneration in a rat model of patellar tendon defect. Collectively, these data show that nestin could function as a marker for TSPCs. This is further strengthened by a previous study showing high levels of nestin expression in TSPCs isolated from human Achilles tendon [56]. Taken together, these studies identify sources for TSPCs including the tendon proper (midsubstance) and peritenon that may contribute towards tendon tissue maintenance, healing, or repair.

\section{Interactions of TSPCs with ECM and Mechanical Loading}

Stem cells cannot function without the signals from their niche. The various niche factors for stem cells include ECM and mechanical stress, as well as oxygen tension, growth factors, and cytokines $[4,40,57,58]$. Considering the surrounding rich ECM, the main niche signals that TSPCs receive may be from those ECM components such as biglycan and fibromodulin [4]. The ECM microenvironment likely plays an important role in TSPC fate that ultimately affects tendon maintenance and repair when injury occurs to the tendon [4]. Alteration of the ECM may lead to tendon pathological conditions, but whether this altered composition of ECM will directly affect the fate of TSPCs remains rather unexplored.

In addition to rich collagen, tendon ECM contains small amounts of proteoglycans (PGs) [2, 35]. Small leucine-rich proteins (SLRPs) are the most abundant PGs present in ten- don and act as the crucial components of ECM, as well as function as an organizer for collagen fibril assembly and regulators of ECM turnover [59, 60]. Decorin and biglycan are the main SLRPs in tendon. SLRPs such as fibromodulin and lumican are also present in tendon. The tendons of decorin/biglycan/fibromodulin-deficient animals are mechanically inferior to normal tendons of wild-type mice $[61,62]$, and the collagen fibers within the tendon become disorganized in the absence of biglycan and fibromodulin [4]. Tendon integrity is impaired in lumican and fibromodulindeficient mice [63]. Alteration of the ECM composition changes the structure of the TSPC niche consequently affecting the fate of TSPCs, which leads to tendon malformation and ossification [4]. Biglycan and fibromodulin are two critical SLRPs that control the fate of TSPCs. This may be mediated in part by modulating bone morphogenic protein (BMP) activity. TSPCs from biglycan and fibromodulin double-knockout mice proliferate faster, form larger colonies, and form bone-like tissues in addition to tendon-like tissues compared to those from wild type (WT) mice which form only tendon-like tissues [4]. The increased sensitivity of TSPCs to BMP-2 in the absence of biglycan and fibromodulin could be a mechanism for altering the fate of TSPCs. The expression of tendon markers Scx and collagen type I is decreased in TSPCs from these knockout mice compared to cells from WT mice. Therefore, the integrity of ECM is important in maintaining the stemness of TSPCs, and the precise regulation of the tenogenic differentiation of TSPCs is essential for the positive outcome of stem cell-based therapy for injured tendons.

In tendon, cell-ECM interactions maintain tissue homeostasis by generating cell signals that affect cell proliferation, differentiation, migration, and adhesion [35]. On the other hand, the ECM plays an important role in disease progression. The tendon ECM is enriched in growth factors and cytokines, and the ECM plays a major role in regulating the local availability of growth factors at a cellular level [64]. The changes of the structure and composition of ECM may disturb the local release of growth factors and cytokines as well as the modulation of cell shape and signaling cascade affecting the cell fate. Aberrant ECM changes including calcification, ossification, and lipid and proteoglycan accumulation are evident in human tendinopathy samples $[65,66]$. The aberrant differentiation of TSPCs to nontenocytes (adipocytes, chondrocytes, and osteoblasts), which produce nontendinous tissues, is suggested as a possible mechanism in the development of tendinopathy due to mechanical overloading placed on the tendon $[30,32,33]$.

An engineered tendon matrix (ETM) from decellularized tendon tissues stimulates rabbit TSPC proliferation and better preserves stemness compared to plastic culture surfaces commonly used in culture, and implantation of ETM-TSPC composite promotes tendon-like tissue formation [67]. The ECM components and/or growth factors may contribute these properties to TSPCs, which are important in tissue engineering applications of such composites in injured tendon repair. A similar study using decellularized collagenous matrix from three different tissues (tendon, bone, and dermis) showed that tendon-derived decellularized matrix 
promotes the tendinous phenotype in human TSPCs and inhibits their osteogenesis, even under osteogenic induction conditions, although all the three matrices support cell adhesion and proliferation [68]. The bone-derived decellularized matrix robustly induces osteogenic differentiation of TSPCs, whereas the dermal skin-derived collagen matrix induces only an intermediate level of osteogenesis. The differential cellular response could be attributed to the differences in the structure and topography but otherwise similar bioactivity of the matrices. The cell shape and alignment also differed in the three matrices; cells adopt an elongated shape and align on the tendon matrix, but not on the dermis matrix. This shows that besides the composition, ECM topographical cues are important in regulating the stem cell fate. This is supported by yet another study, which indicated that the culture of human TSPCs in an aligned nanofiber scaffold promotes tenogenic commitment, but in a random scaffold enhances osteogenic differentiation [42]. Compared to embryonic stem cell-mesenchymal stromal cells, TSPCs combined with the decellularized matrix also show more improvement in the structural and biomechanical properties of regenerated tendons in vivo [69]. In short, ECM components provide niche signals to TSPCs and play a significant role in deciding their fate depending on the changes in ECM composition and topographical cues.

The precise function of TSPCs in vivo is not well defined yet, and comparison studies with tenocytes are rare. Tendons have poor regenerative capacity as demonstrated by the inferior quality of tissues following injury or chronic degeneration $[70,71]$. Therefore, it is conceivable that TSPCs alone may not be able to functionally restore the damaged tissues, although TSPCs promote functional repair of tendon tissues [72-77].

It is well known that mechanical loads play a major role in tendon development, homeostasis, pathology, and injury healing. These forces are translated into biochemical signals by molecules possessing mechanotransduction capabilities which activate and control key cellular processes of tendon $[11,78]$. Normal mechanical loads are essential for appropriate tendon development and maintenance, because such loads like moderate loading patterns induce cellular anabolic adaptation of tendon [78-80]. On the other hand, abnormal mechanical loads cause pathological conditions (e.g. tendinopathy) in tendon by inducing dominant catabolic responses in tendon cells [14,81-85]. Tendon cells respond to mechanical loads and modulate ECM via various mechanisms/pathways which have been extensively investigated using both in vitro and in vivo loading models (e.g., [86-88]).

TSPCs are capable of altering the tendon ECM in response to modifications of the loading environments. Also, the multidifferentiation potential of TSPCs allow them to differentially respond to altering mechanical loads. For example, an in vitro study showed that a uniaxial cyclic mechanical stretching of patellar and Achilles TSPCs from mice at moderate levels ( $4 \%$ elongation, $0.5 \mathrm{~Hz}$ for $12 \mathrm{hrs}$ ) increases proliferation and collagen type I gene expression without affecting the gene expressions of PPAR $\gamma$ (a marker for adipocytes), collagen type II, SOX-9 (markers for chon- drocytes), and Runx2 (a marker osteocytes) [30]. Similarly, mechanical loading in the form of moderate treadmill running in mice increases the proliferation of TSPCs and TSPC-related cellular production of collagen [57].

However, mechanical stretching of mouse TSPCs at an excessive level ( $8 \%$ elongation) increases the gene expression of PPAR $\gamma$, collagen type II, SOX-9, and Runx2 [30]. A further study showed that a $4 \%$ stretching of TSPCs increases the expression of tenocyte-related genes (collagen I and TNMD) while $8 \%$ stretching increased the expression of both tenocyte and non-tenocyte-related genes (LPL, SOX-9, and Runx2) in TSPCs but not in tenocytes [33]. The increase in both tenocyte and nontenocyte-related gene expression under $8 \%$ stretching may be due to the fact that the TSPC population is heterogeneous, meaning that individual TSPC may have different levels of threshold in response to mechanical loading and as a result, they respond differently in their gene expression. Similar results were obtained when the study was performed in vivo using moderate and intensive treadmill running to apply low and excess mechanical loading, respectively [33]. Although the results from cellular and tissue levels are presented only at gene expression levels, it may indicate that mechanical overloading may prime TSPCs to undergo nontenocyte differentiation. Further studies are warranted to link the nontenogenic differentiation of TSPCs and degenerative changes in tendinopathy.

Also, in another study, mechanical stretching at $4 \%$ and $8 \%(0.5 \mathrm{~Hz}$ for $4 \mathrm{hrs})$ increased BMP-2 expression at gene and protein levels in rat TSPCs [31]. BMP-2 increased osteogenic differentiation of TSPCs as indicated by ALP activity and calcium nodule formation. The observation of osteogenic differentiation at $4 \%$ is in contrast with the previous findings [30, 33], possibly due to the differences in the stretching regimen, species difference, and culture conditions. Involvement of BMP-2 in the pathogenesis of tendinopathy has been suggested previously based on the reported observations that chondrocyte phenotype and ectopic ossification are present in calcifying tendinopathy and based on the expression of BMP-2 protein at those sites [89-92]. Moreover, the addition of BMP-2 to human TSPCs in culture decreased cell proliferation and induced osteogenic differentiation [93]. Higher BMP-2 receptor expression and BMP-2-induced osteogenic differentiation of rat TSPCs compared to BMSCs have been reported [94]. Taken together, these studies indicate that the activation of BMP-2 expression in TSPCs during tendon overuse might provide a possible explanation for ectopic calcification in calcifying tendinopathy. Overall, the data indicate that moderate mechanical loads are beneficial for maintaining tendon homeostasis, but mechanical overloading may contribute to degenerative changes in tendon, with TSPCs playing a major role.

\section{Conclusion}

TSPCs have been identified in both the tendon proper and peritenon in tendon that may have both vascular and nonvascular origins. However, how TSPCs from different locations contribute to tendon maintenance, repair, and 
tendinopathy remains to be better understood. This is indeed a challenging task now considering the lack of definitive genetic lineage tracing and specific markers for TSPC identity, functions, and biological characterization. To this end, advanced studies using state-of-the-art novel approaches, including genetic models, genetic lineage tracing, and single-cell transcription profiling to identify the heterogeneity of TSPCs, need to be conducted.

TSPCs reside in tendons and are constantly subjected to mechanical loading due to the fact that tendons like Achilles and patellar are load-bearing tissues. Consequently, the function of TSPCs is regulated by ECM composition, organization, and mechanical loads. Therefore, further investigations are necessary to understand the crosstalk among TSPCs, ECM, and mechanical loads, and also those signaling pathways involved, so that tendon physiology and pathophysiology can be better understood. The findings from these investigations will surely aid in devising new yet effective tissue engineering approaches to regenerate injured tendons and also developing novel treatment strategies to manage tendinopathy, a prevalent tendon disorder commonly seen in both athletic and general populations.

\section{Abbreviations}

ALP: $\quad$ Alkaline phosphatase

BMP-2: $\quad$ Bone morphogenetic protein-2

BMSCs: Bone marrow mesenchymal stem cells

Col I: $\quad$ Collagen type I

Comp: Collagen oligomeric matrix protein

ECM: $\quad$ Extracellular matrix

ETM: $\quad$ Engineered tendon matrix

FM: $\quad$ Fascicular matrix

GAG: Glycosaminoglycans

HSR: Heavy slow resistance

IFM: Interfascicular matrix

LPL: $\quad$ Lipoprotein lipase

LRC: Label-retaining cells

NSAIDs: Nonsteroidal anti-inflammatory drugs

NCSCs: Neuronal crest stem cells

Oct-4: Octamer-binding transcription factor 4

PGs: $\quad$ Proteoglycans

PPAR $\gamma$ : Peroxisome proliferator-activated receptor $\gamma$

PRP: $\quad$ Platelet-rich plasma

Runx2: Runt-related transcription factor 2

Scx: $\quad$ Scleraxis

SLRPs: $\quad$ Small leucine-rich proteins

Smad8: $\quad$ Mothers against decapentaplegic homolog 8

SOX-9: $\quad$ Sex-determining region SRY

SSEA-1/4: Stage-specific embryonic antigen 1/4

Thbs4: Thrombospondin 4

TNMD: Tenomodulin

TSPCs: Tendon stem/progenitor cells.

\section{Conflicts of Interest}

The authors declare that there are no conflicts of interest regarding the publication of this paper.

\section{Authors' Contributions}

Chuanxin Zhang and Jun Zhu contributed equally to this review.

\section{Acknowledgments}

This work was supported by grants from the National Natural Science Foundation of China (8170090144) and the National Institutes of Health, USA (AR065949). The authors thank Dr. Juntao Ji for preparing the illustration (Figure 1).

\section{References}

[1] G. Nourissat, F. Berenbaum, and D. Duprez, "Tendon injury: from biology to tendon repair," Nature Reviews Rheumatology, vol. 11, no. 4, pp. 223-233, 2015.

[2] C. T. Thorpe and H. R. C. Screen, "Tendon structure and composition," in Metabolic Influences on Risk for Tendon Disorders, P. Ackermann and D. Hart, Eds., vol. 920 of Advances in Experimental Medicine and Biology, pp. 3-10, Springer, Cham, 2016.

[3] P. Kannus, "Structure of the tendon connective tissue," Scandinavian Journal of Medicine \& Science in Sports, vol. 10, no. 6, pp. 312-320, 2000.

[4] Y. Bi, D. Ehirchiou, T. M. Kilts et al., "Identification of tendon stem/progenitor cells and the role of the extracellular matrix in their niche," Nature Medicine, vol. 13, no. 10, pp. 1219-1227, 2007.

[5] J. Zhang and J. H. C. Wang, "Characterization of differential properties of rabbit tendon stem cells and tenocytes," BMC Musculoskeletal Disorders, vol. 11, no. 1, p. 10, 2010.

[6] E. Zelzer, E. Blitz, M. L. Killian, and S. Thomopoulos, "Tendon-to-bone attachment: from development to maturity," Birth Defects Research Part C: Embryo Today: Reviews, vol. 102, no. 1, pp. 101-112, 2014.

[7] H. Tempfer, A. Wagner, R. Gehwolf et al., "Perivascular cells of the supraspinatus tendon express both tendon- and stem cellrelated markers," Histochemistry and Cell Biology, vol. 131, no. 6, pp. 733-741, 2009.

[8] M. J. Mienaltowski, S. M. Adams, and D. E. Birk, "Regional differences in stem cell/progenitor cell populations from the mouse Achilles tendon," Tissue Engineering Part A, vol. 19, no. 1-2, pp. 199-210, 2013.

[9] Q. Tan, P. P. Y. Lui, and Y. W. Lee, "In vivo identity of tendon stem cells and the roles of stem cells in tendon healing," Stem Cells and Development, vol. 22, no. 23, pp. 3128-3140, 2013.

[10] J. H. C. Wang, Q. Guo, and B. Li, “Tendon biomechanics and mechanobiology - a minireview of basic concepts and recent advancements," Journal of Hand Therapy, vol. 25, no. 2, pp. 133-141, 2012.

[11] M. Lavagnino, M. E. Wall, D. Little, A. J. Banes, F. Guilak, and S. P. Arnoczky, "Tendon mechanobiology: current knowledge and future research opportunities," Journal of Orthopaedic Research, vol. 33, no. 6, pp. 813-822, 2015.

[12] C. N. Maganaris, M. V. Narici, L. C. Almekinders, and N. Maffulli, "Biomechanics and pathophysiology of overuse tendon injuries: ideas on insertional tendinopathy," Sports Medicine, vol. 34, no. 14, pp. 1005-1017, 2004. 
[13] M. Kjaer, "Role of extracellular matrix in adaptation of tendon and skeletal muscle to mechanical loading," Physiological Reviews, vol. 84, no. 2, pp. 649-698, 2004.

[14] D. Docheva, S. A. Müller, M. Majewski, and C. H. Evans, "Biologics for tendon repair," Advanced Drug Delivery Reviews, vol. 84, pp. 222-239, 2015.

[15] J. F. Kaux, B. Forthomme, C. L. Goff, J. M. Crielaard, and J. L. Croisier, "Current opinions on tendinopathy," Journal of Sports Science and Medicine, vol. 10, no. 2, pp. 238-253, 2011.

[16] J. C. Patterson-Kane and T. Rich, "Achilles tendon injuries in elite athletes: lessons in pathophysiology from their equine counterparts," ILAR Journal, vol. 55, no. 1, pp. 86-99, 2014.

[17] B. J. F. Dean, S. G. Dakin, N. L. Millar, and A. J. Carr, "Review: emerging concepts in the pathogenesis of tendinopathy," The Surgeon, vol. 15, no. 6, pp. 349-354, 2017.

[18] J. L. Y. Leung and J. F. Griffith, "Sonography of chronic Achilles tendinopathy: a case-control study," Journal of Clinical Ultrasound, vol. 36, no. 1, pp. 27-32, 2008.

[19] U. G. Longo, M. Ronga, and N. Maffulli, "Achilles tendinopathy," Sports Medicine and Arthroscopy Review, vol. 17, no. 2, pp. 112-126, 2009.

[20] J. L. Cook and C. R. Purdam, "Is tendon pathology a continuum? A pathology model to explain the clinical presentation of load-induced tendinopathy," British Journal of Sports Medicine, vol. 43, no. 6, pp. 409-416, 2009.

[21] J. L. Cook, E. Rio, C. R. Purdam, and S. I. Docking, "Revisiting the continuum model of tendon pathology: what is its merit in clinical practice and research?," British Journal of Sports Medicine, vol. 50, no. 19, pp. 1187-1191, 2016.

[22] N. L. Millar, G. A. C. Murrell, and I. B. McInnes, "Inflammatory mechanisms in tendinopathy-towards translation," Nature Reviews Rheumatology, vol. 13, no. 2, pp. 110-122, 2017.

[23] M. Benjamin and J. R. Ralphs, "The cell and developmental biology of tendons and ligaments," International Review of Cytology, vol. 196, pp. 85-130, 2000.

[24] S. A. Fenwick, B. L. Hazleman, and G. P. Riley, "The vasculature and its role in the damaged and healing tendon," Arthritis Research, vol. 4, no. 4, pp. 252-260, 2002.

[25] G. Riley, "The pathogenesis of tendinopathy. A molecular perspective," Rheumatology, vol. 43, no. 2, pp. 131-142, 2004.

[26] Y. Xu and G. A. C. Murrell, "The basic science of tendinopathy," Clinical Orthopaedics and Related Research, vol. 466, no. 7, pp. 1528-1538, 2008.

[27] G. Riley, "Tendinopathy-from basic science to treatment," Nature Clinical Practice Rheumatology, vol. 4, no. 2, pp. 82$89,2008$.

[28] N. Maffulli, U. G. Longo, and V. Denaro, "Novel approaches for the management of tendinopathy," The Journal of Bone and Joint Surgery, vol. 92, no. 15, pp. 2604-2613, 2010.

[29] M. Kongsgaard, K. Qvortrup, J. Larsen et al., "Fibril morphology and tendon mechanical properties in patellar tendinopathy: effects of heavy slow resistance training," The American Journal of Sports Medicine, vol. 38, no. 4, pp. 749-756, 2010.

[30] J. Zhang and J. H. C. Wang, "Mechanobiological response of tendon stem cells: implications of tendon homeostasis and pathogenesis of tendinopathy," Journal of Orthopaedic Research, vol. 28, no. 5, pp. 639-643, 2010.

[31] Y. F. Rui, P. P. Y. Lui, M. Ni, L. S. Chan, Y. W. Lee, and K. M. Chan, "Mechanical loading increased BMP-2 expression which promoted osteogenic differentiation of tendon-derived stem cells," Journal of Orthopaedic Research, vol. 29, no. 3, pp. 390-396, 2011.

[32] Y. F. Rui, P. P. Lui, L. S. Chan, K. M. Chan, S. C. Fu, and G. Li, "Does erroneous differentiation of tendon-derived stem cells contribute to the pathogenesis of calcifying tendinopathy?," Chinese Medical Journal, vol. 124, no. 4, pp. 606-610, 2011.

[33] J. Zhang and J. H. C. Wang, "The effects of mechanical loading on tendons - an in vivo and in vitro model study," PLoS One, vol. 8, no. 8, article e71740, 2013.

[34] N. A. Dyment and J. L. Galloway, "Regenerative biology of tendon: mechanisms for renewal and repair," Current Molecular Biology Reports, vol. 1, no. 3, pp. 124-131, 2015.

[35] H. R. C. Screen, D. E. Berk, K. E. Kadler, F. Ramirez, and M. F. Young, "Tendon functional extracellular matrix," Journal of Orthopaedic Research, vol. 33, no. 6, pp. 793-799, 2015.

[36] D. Docheva, E. B. Hunziker, R. Fassler, and O. Brandau, "Tenomodulin is necessary for tenocyte proliferation and tendon maturation," Molecular and Cellular Biology, vol. 25, no. 2, pp. 699-705, 2005.

[37] R. Salingcarnboriboon, H. Yoshitake, K. Tsuji et al., "Establishment of tendon-derived cell lines exhibiting pluripotent mesenchymal stem cell-like property," Experimental Cell Research, vol. 287, no. 2, pp. 289-300, 2003.

[38] M. de Mos, W. J. L. M. Koevoet, H. Jahr et al., "Intrinsic differentiation potential of adolescent human tendon tissue: an invitro cell differentiation study," BMC Musculoskeletal Disorders, vol. 8, no. 1, p. 16, 2007.

[39] Y. F. Rui, P. P. Y. Lui, G. Li, S. C. Fu, Y. W. Lee, and K. M. Chan, "Isolation and characterization of multipotent rat tendon-derived stem cells," Tissue Engineering Part A, vol. 16, no. 5, pp. 1549-1558, 2010.

[40] P. P. Y. Lui, "Identity of tendon stem cells - how much do we know?," Journal of Cellular and Molecular Medicine, vol. 17, no. 1, pp. 55-64, 2013.

[41] P. P. Y. Lui and K. M. Chan, "Tendon-derived stem cells (TDSCs): from basic science to potential roles in tendon pathology and tissue engineering applications," Stem Cell Reviews and Reports, vol. 7, no. 4, pp. 883-897, 2011.

[42] Z. Yin, X. Chen, J. L. Chen et al., "The regulation of tendon stem cell differentiation by the alignment of nanofibers," Biomaterials, vol. 31, no. 8, pp. 2163-2175, 2010.

[43] Z. Zhou, T. Akinbiyi, L. Xu et al., "Tendon-derived stem/progenitor cell aging: defective self-renewal and altered fate," Aging Cell, vol. 9, no. 5, pp. 911-915, 2010.

[44] P. P. Lui, Y. F. Rui, and M. Ni, "Comparison of potentials of stem cells isolated from tendon and bone marrow for musculoskeletal tissue engineering," in 57th Annual Meeting of the Orthopaedic Research Society, Long Beach, CA, 2011.

[45] M. T. Harris, D. L. Butler, G. P. Boivin, J. B. Florer, E. J. Schantz, and R. J. Wenstrup, "Mesenchymal stem cells used for rabbit tendon repair can form ectopic bone and express alkaline phosphatase activity in constructs," Journal of Orthopaedic Research, vol. 22, no. 5, pp. 998-1003, 2004.

[46] C. T. Thorpe, P. D. Clegg, and H. L. Birch, "A review of tendon injury: why is the equine superficial digital flexor tendon most at risk?," Equine Veterinary Journal, vol. 42, no. 2, pp. 174180, 2010.

[47] E. M. Spiesz, C. T. Thorpe, S. Chaudhry et al., "Tendon extracellular matrix damage, degradation and inflammation in response to in vitro overload exercise," Journal of Orthopaedic Research, vol. 33, no. 6, pp. 889-897, 2015. 
[48] G. Yang, B. B. Rothrauff, and R. S. Tuan, "Tendon and ligament regeneration and repair: clinical relevance and developmental paradigm," Birth Defects Research Part C: Embryo Today, vol. 99, no. 3, pp. 203-222, 2013.

[49] M. J. Mienaltowski, A. Cánovas, V. A. Fates et al., "Transcriptome profiles of isolated murine Achilles tendon properand peritenon-derived progenitor cells," Journal of Orthopaedic Research, vol. 37, no. 6, pp. 1409-1418, 2019.

[50] W. Xu, Y. Sun, J. Zhang et al., "Perivascular-derived stem cells with neural crest characteristics are involved in tendon repair," Stem Cells and Development, vol. 24, no. 7, pp. 857-868, 2015.

[51] N. A. Dyment, Y. Hagiwara, B. G. Matthews, Y. Li, I. Kalajzic, and D. W. Rowe, "Lineage tracing of resident tendon progenitor cells during growth and natural healing," PLoS One, vol. 9, no. 4, article e96113, 2014.

[52] Z. Yin, J. J. Hu, L. Yang et al., "Single-cell analysis reveals a nestin $^{+}$tendon stem/progenitor cell population with strong tenogenic potentiality," Science Advances, vol. 2, no. 11, article e1600874, 2016.

[53] C. Wiese, A. Rolletschek, G. Kania et al., "Nestin expression-a property of multi-lineage progenitor cells?," Cellular and Molecular Life Sciences, vol. 61, no. 19-20, pp. 2510-2522, 2004.

[54] K. Rizzoti, "Adult pituitary progenitors/stem cells: from in vitro characterization to in vivo function," The European Journal of Neuroscience, vol. 32, no. 12, pp. 2053-2062, 2010.

[55] D. Park, A. P. Xiang, F. F. Mao et al., "Nestin is required for the proper self-renewal of neural stem cells," Stem Cells, vol. 28, no. 12, pp. 2162-2171, 2010.

[56] C. Popov, M. Burggraf, L. Kreja, A. Ignatius, M. Schieker, and D. Docheva, "Mechanical stimulation of human tendon stem/progenitor cells results in upregulation of matrix proteins, integrins and MMPs, and activation of p38 and ERK1/2 kinases," BMC Molecular Biology, vol. 16, no. 1, p. 6, 2015.

[57] J. Zhang, T. Pan, Y. Liu, and J. H. C. Wang, "Mouse treadmill running enhances tendons by expanding the pool of tendon stem cells (TSCs) and TSC-related cellular production of collagen," Journal of Orthopaedic Research, vol. 28, no. 9, pp. 1178$1183,2010$.

[58] W. Y. W. Lee, P. P. Y. Lui, and Y. F. Rui, "Hypoxia-mediated efficient expansion of human tendon-derived stem cells in vitro," Tissue Engineering Part A, vol. 18, no. 5-6, pp. 484498, 2012.

[59] J. H. Yoon and J. Halper, "Tendon proteoglycans: biochemistry and function," Journal of Musculoskeletal \& Neuronal Interactions, vol. 5, no. 1, pp. 22-34, 2005.

[60] C. T. Thorpe, H. L. Birch, P. D. Clegg, and H. R. C. Screen, "The role of the non-collagenous matrix in tendon function," International Journal of Experimental Pathology, vol. 94, no. 4, pp. 248-259, 2013.

[61] M. F. Young, Y. Bi, L. Ameye, and X. D. Chen, "Biglycan knockout mice: new models for musculoskeletal diseases," Glycoconjugate Journal, vol. 19, no. 4/5, pp. 257-262, 2002.

[62] L. M. Dourte, L. Pathmanathan, A. F. Jawad et al., "Influence of decorin on the mechanical, compositional, and structural properties of the mouse patellar tendon," Journal of Biomechanical Engineering, vol. 134, no. 3, article 031005, 2012.

[63] K. J. Jepsen, F. Wu, J. H. Peragallo et al., "A syndrome of joint laxity and impaired tendon integrity in lumican- and fibromodulin-deficient mice," The Journal of Biological Chemistry, vol. 277, no. 38, pp. 35532-35540, 2002.
[64] G. S. Schultz and A. Wysocki, "Interactions between extracellular matrix and growth factors in wound healing," Wound Repair and Regeneration, vol. 17, no. 2, pp. 153-162, 2009.

[65] P. Kannus and L. Jozsa, "Histopathological changes preceding spontaneous rupture of a tendon. A controlled study of 891 patients," The Journal of Bone and Joint Surgery, vol. 73, no. 10, pp. 1507-1525, 1991.

[66] S. C. Fu, K. M. Chan, and C. G. Rolf, "Increased deposition of sulfated glycosaminoglycans in human patellar tendinopathy," Clinical Journal of Sport Medicine, vol. 17, no. 2, pp. 129-134, 2007.

[67] J. Zhang, B. Li, and J. H. C. Wang, "The role of engineered tendon matrix in the stemness of tendon stem cells in vitro and the promotion of tendon-like tissue formation in vivo," Biomaterials, vol. 32, no. 29, pp. 6972-6981, 2011.

[68] Z. Yin, X. Chen, T. Zhu et al., "The effect of decellularized matrices on human tendon stem/progenitor cell differentiation and tendon repair," Acta Biomaterialia, vol. 9, no. 12, pp. 9317-9329, 2013.

[69] H. Song, Z. Yin, T. Wu et al., "Enhanced effect of tendon stem/progenitor cells combined with tendon-derived decellularized extracellular matrix on tendon regeneration," Cell Transplantation, vol. 27, no. 11, pp. 1634-1643, 2018.

[70] H. Miyashita, M. Ochi, and Y. Ikuta, "Histological and biomechanical observations of the rabbit patellar tendon after removal of its central one-third," Archives of Orthopaedic and Trauma Surgery, vol. 116, no. 8, pp. 454-462, 1997.

[71] M. Järvinen, L. Józsa, P. Kannus, T. L. N. Järvinen, M. Kvist, and W. Leadbetter, "Histopathological findings in chronic tendon disorders," Scandinavian Journal of Medicine \& Science in Sports, vol. 7, no. 2, pp. 86-95, 1997.

[72] M. Ni, P. P. Y. Lui, Y. F. Rui et al., “Tendon-derived stem cells (TDSCs) promote tendon repair in a rat patellar tendon window defect model," Journal of Orthopaedic Research, vol. 30, no. 4, pp. 613-619, 2012.

[73] C. Tan, P. P. Y. Lui, Y. W. Lee, and Y. M. Wong, "Scx-transduced tendon-derived stem cells (tdscs) promoted better tendon repair compared to mock-transduced cells in a rat patellar tendon window injury model," PLoS One, vol. 9, no. 5, article e97453, 2014.

[74] L. Chen, J. P. Liu, K. L. Tang et al., "Tendon derived stem cells promote platelet-rich plasma healing in collagenase-induced rat Achilles tendinopathy," Cellular Physiology and Biochemistry, vol. 34, no. 6, pp. 2153-2168, 2014.

[75] D. Jiang, B. Xu, M. Yang, Z. Zhao, Y. Zhang, and Z. Li, "Efficacy of tendon stem cells in fibroblast-derived matrix for tendon tissue engineering," Cytotherapy, vol. 16, no. 5, pp. 662-673, 2014.

[76] P. P. Y. Lui, O. T. Wong, and Y. W. Lee, "Transplantation of tendon-derived stem cells pre-treated with connective tissue growth factor and ascorbic acid in vitro promoted better tendon repair in a patellar tendon window injury rat model," Cytotherapy, vol. 18, no. 1, pp. 99-112, 2016.

[77] I. Komatsu, J. H. C. Wang, K. Iwasaki, T. Shimizu, and T. Okano, "The effect of tendon stem/progenitor cell (TSC) sheet on the early tendon healing in a rat Achilles tendon injury model," Acta Biomaterialia, vol. 42, pp. 136-146, 2016.

[78] M. T. Galloway, A. L. Lalley, and J. T. Shearn, "The role of mechanical loading in tendon development, maintenance, injury, and repair," The Journal of Bone and Joint Surgery, vol. 95, no. 17, pp. 1620-1628, 2013. 
[79] K. M. Heinemeier and M. Kjaer, "In vivo investigation of tendon responses to mechanical loading," Journal of Musculoskeletal \& Neuronal Interactions, vol. 11, no. 2, pp. 115-123, 2011.

[80] K. M. Heinemeier, D. Skovgaard, M. L. Bayer et al., "Uphill running improves rat Achilles tendon tissue mechanical properties and alters gene expression without inducing pathological changes," Journal of Applied Physiology (Bethesda, MD: 1985), vol. 113, no. 5, pp. 827-836, 2012.

[81] J. G. Snedeker and J. Foolen, "Tendon injury and repair-a perspective on the basic mechanisms of tendon disease and future clinical therapy," Acta Biomaterialia, vol. 63, pp. 18$36,2017$.

[82] L. J. Soslowsky, S. Thomopoulos, S. Tun et al., "Neer Award 1999. Overuse activity injures the supraspinatus tendon in an animal model: a histologic and biomechanical study," Journal of Shoulder and Elbow Surgery, vol. 9, no. 2, pp. 79-84, 2000.

[83] J. H. C. Wang, "Mechanobiology of tendon," Journal of Biomechanics, vol. 39, no. 9, pp. 1563-1582, 2006.

[84] M. Attia, A. Scott, A. Duchesnay et al., "Alterations of overused supraspinatus tendon: a possible role of glycosaminoglycans and HARP/pleiotrophin in early tendon pathology," Journal of Orthopaedic Research, vol. 30, no. 1, pp. 61-71, 2012.

[85] T. W. Herod and S. P. Veres, "Development of overuse tendinopathy: a new descriptive model for the initiation of tendon damage during cyclic loading," Journal of Orthopaedic Research, vol. 36, no. 1, pp. 467-476, 2018.

[86] R. C. Dirks and S. J. Warden, "Models for the study of tendinopathy," Journal of Musculoskeletal \& Neuronal Interactions, vol. 11, no. 2, pp. 141-149, 2011.

[87] T. Wang, P. Chen, M. Zheng et al., "In vitro loading models for tendon mechanobiology," Journal of Orthopaedic Research, vol. 36, no. 2, pp. 566-575, 2018.

[88] B. P. Thampatty and J. H. C. Wang, "Mechanobiology of young and aging tendons: in vivo studies with treadmill running," Journal of Orthopaedic Research, vol. 36, no. 2, pp. 557-565, 2018.

[89] S. Fenwick, R. Harrall, R. Hackney et al., "Endochondral ossification in Achilles and patella tendinopathy," Rheumatology, vol. 41, no. 4, pp. 474-476, 2002.

[90] N. Maffulli, J. Reaper, S. W. B. Ewen, S. W. Waterston, and V. Barrass, "Chondral metaplasia in calcific insertional tendinopathy of the Achilles tendon," Clinical Journal of Sport Medicine, vol. 16, no. 4, pp. 329-334, 2006.

[91] P. P. Y. Lui, S. C. Fu, L. S. Chan, L. K. Hung, and K. M. Chan, "Chondrocyte phenotype and ectopic ossification in collagenase-induced tendon degeneration," Journal of Histochemistry \& Cytochemistry, vol. 57, no. 2, pp. 91-100, 2009.

[92] P. P. Y. Lui, L. S. Chan, Y. C. Cheuk, Y. W. Lee, and K. M. Chan, "Expression of bone morphogenetic protein-2 in the chondrogenic and ossifying sites of calcific tendinopathy and traumatic tendon injury rat models," Journal of Orthopaedic Surgery and Research, vol. 4, no. 1, p. 27, 2009.
[93] J. Zhang and J. H. C. Wang, "BMP-2 mediates PGE $_{2}$-induced reduction of proliferation and osteogenic differentiation of human tendon stem cells," Journal of Orthopaedic Research, vol. 30, no. 1, pp. 47-52, 2012.

[94] Y. F. Rui, P. P. Y. Lui, Y. W. Lee, and K. M. Chan, "Higher BMP receptor expression and BMP-2-induced osteogenic differentiation in tendon-derived stem cells compared with bonemarrow-derived mesenchymal stem cells," International Orthopaedics, vol. 36, no. 5, pp. 1099-1107, 2012. 


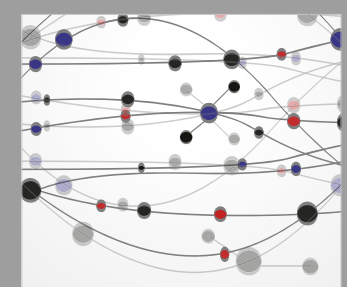

The Scientific World Journal
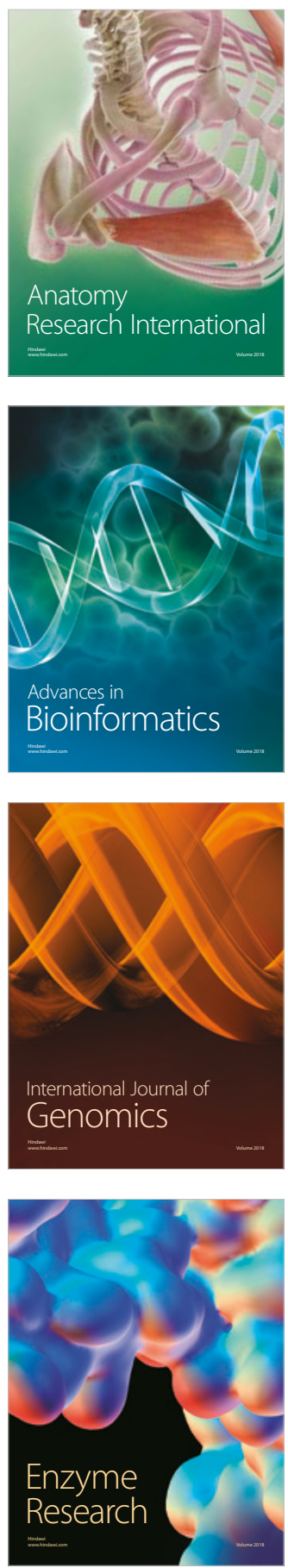
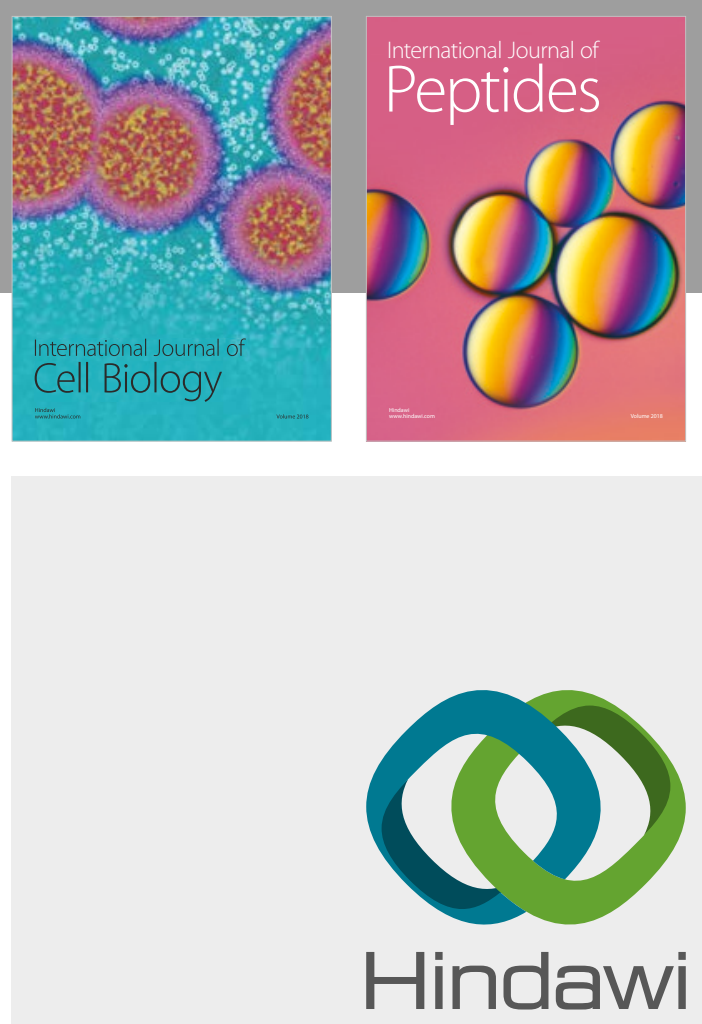

Submit your manuscripts at

www.hindawi.com
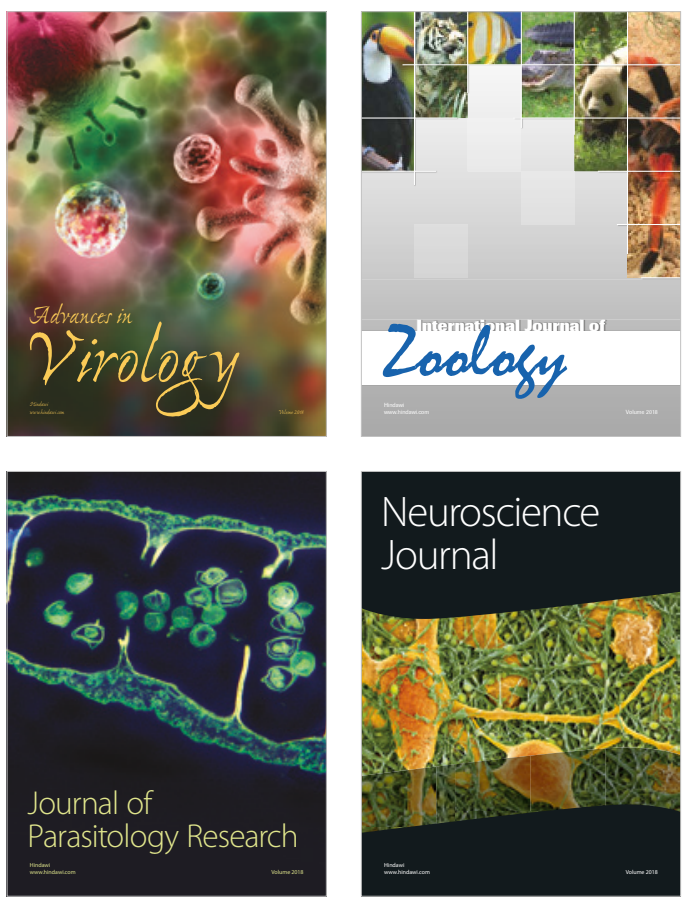
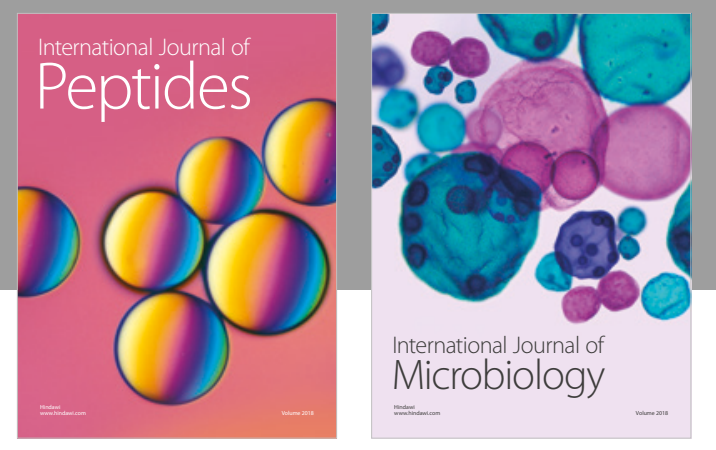

nternational Journal of Microbiology
Journal of
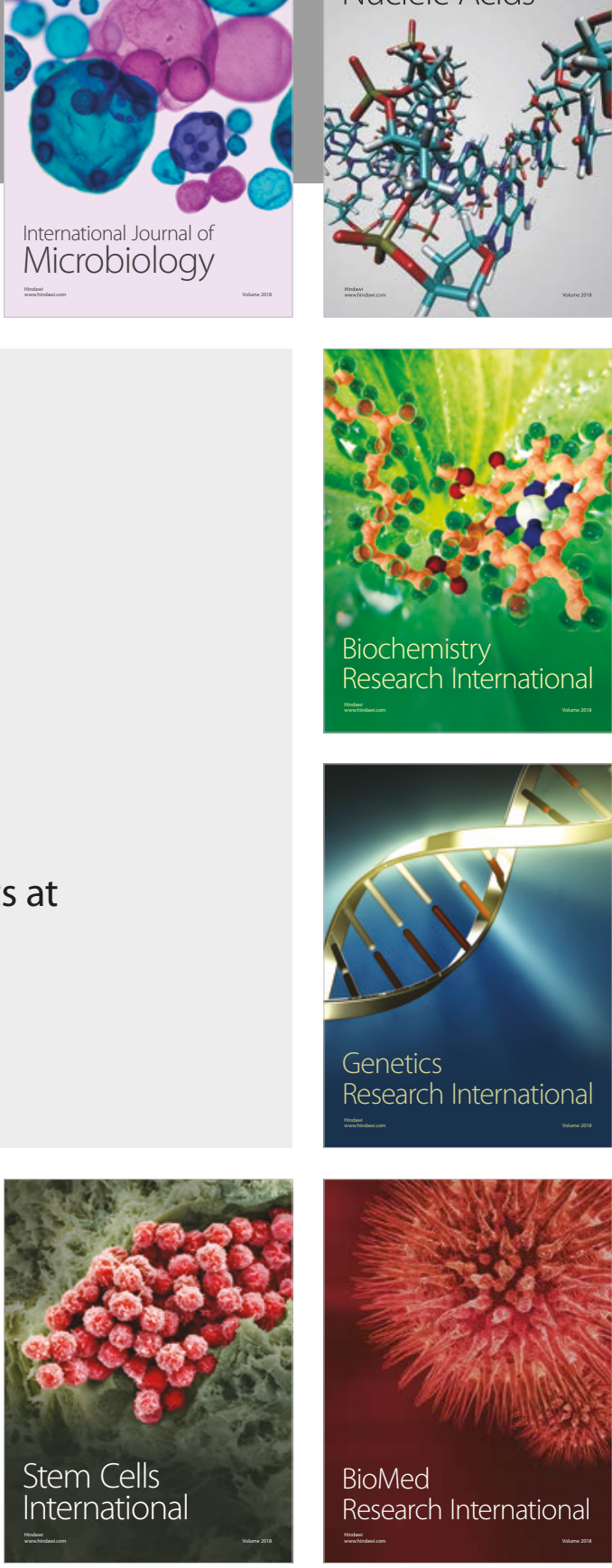
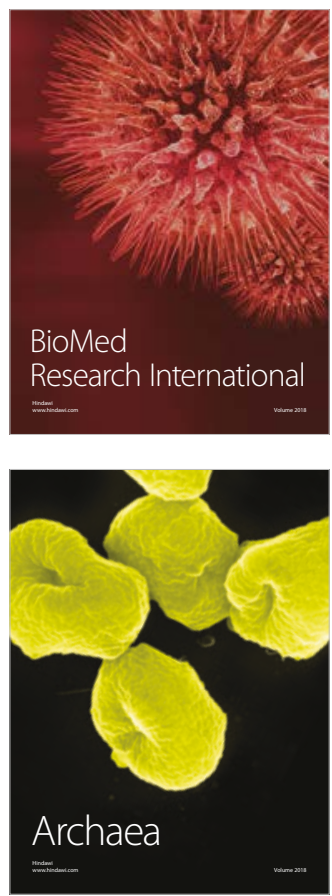